\title{
DeRMATOSES EM PACIENTES COM AIDS: ESTUDO DE 55 CASOS. UBERLÂNDIA, MG, BRASIL
}

\author{
Mabel Duarte Alves Gomides, Alceu luiz Camargo Villela Berbert*, Sônia Antunes de Oliveira Mantese, \\ Adenir Rocha, Marcelo Simão Ferreira, Aercio S. Borges \\ Trabalho realizado no Serviço de Dermatologia da Universidade Federal de Uberlândia (UFU) -MG
}

RESUMO - Foram estudados 55 pacientes com a síndrome da imunodeficiência adquirida (Aids), triados ao serviço de Dermatologia, apresentando doenças cutâneo-mucosas, algumas de caráter oportunista. As dermatoses são freqüentes nestes doentes e, às vezes, de difícil diagnóstico pelo caráter atípico das lesões.

Objetivos. Analisar a freqüência e apresentação clínica das dermatoses relacionadas à Aids.

Métodos. Cinqüenta e cinco pacientes com Aids e lesões tegumentares foram estudados, de modo transversal, no Serviço de Dermatologia da Universidade Federal de Uberlândia, de 1995 a 1997. Foram realizadas biopsias e culturas diversas para elucidação diagnóstica.

RESULTADOS. Foram encontradas I I 6 dermatoses, com predomí- nio das fúngicas $(78 \%)$, seguidas pelas virais (40\%), eritêmatoescamosas (27\%), pápulo-pruríticas (18\%), causadas por drogas (10\%), neoplásicas (9\%) e outras afecções cutâneo-mucosas (7\%). A maioria dos pacientes apresentou mais de uma dermatose (67\%).

CONCLUSÖEs. Confirmou-se a maior freqüência de dermatoses fúngicas, seguidas pelas virais, na Aids. Observou-se a necessidade de propedêutica bem elaborada para 0 diagnóstico preciso das dermatoses, devido à sua apresentação atípica em grande número de doentes. 0 exame dermatológico e a biopsia das lesões tiveram grande relevância na suspeita do diagnóstico de Aids.

UnITERMOS: Aids. Afecções cutâneo-mucosas.

\section{INTRODUÇÃO}

As doenças tegumentares têm sido descritas com relativa freqüência em pacientes infectados pelo vírus da imunodeficiência humana (HIV). Tais afecções têm sido detectadas em mais de 90\% dos indivíduos VIH-positivos ${ }^{\text {I-II, muitas }}$ vezes constituindo os primeiros sinais clínicos da doença ${ }^{7,17}$. As manifestações cutâneo-mucosas podem aparecer mesmo antes da síndrome propriamente dita, com características que sugerem a soropositividade ${ }^{12}$, principalmente quando se acompanham de outros sinais clínicos e de dados epidemiológicos suspeitos ${ }^{13}$.

*Correspondência:

Alceu Luiz Camargo V. Berbert. Rua Dimas Moreira de Sá, 2.205 CEP: 38406-281 - Uberlândia - MG Tel.: (34) 9979-0070
Com freqüência, as doenças tegumentares associadas à infecção pelo HIV apresentam-se de forma exuberante e atípica 14,15,17,19. Sua prevalência tem sido semelhante em pacientes com Aids ou simplesmente HIVpositivos $^{6,15}$. As lesões dermatológicas podem inclusive ser usadas para monitorizar a progressão da infecção pelo HIV 5,8,16.

As dermatoses nos portadores do HIV são de natureza diversa, podendo ter caráter neoplásico, infeccioso ou inflamatório.

$\mathrm{Na}$ literatura nacional foram encontrados poucos trabalhos analisando a prevalência das dermatoses em indivíduos infectados pelo HIV.

Visando contribuir para o conhecimento das afecções cutâneo-mucosas em pacientes com Aids em nosso país, estudou-se, transversalmente, a freqüência das mesmas no Serviço de Dermatologia do Hospital de Clínicas da Universidade Federal de Uberlândia (HC-UFU), Minas Gerais.
Métodos

Foi realizado estudo transversal de 55 pacientes encaminhados do Serviço de Moléstias Infecciosas e do Pronto Socorro do HC-UFU, no período de agosto de 1995 a dezembro de 1997. O protocolo utilizado constava de dados de identificação, dois testes ELISA confirmando a presença do $H I V$, caracterização de grupos de risco, antecedentes patológicos referentes à Aids coletados diretamente do paciente e/ou de seu prontuário, descrição pormenorizada das lesões dermatológicas e exames complementares. Odiagnóstico das dermatoses foi baseado no aspecto clínico e confirmado, quando necessário, pelos seguintes procedimentos: biopsias das lesões com envio de material para exame histopatológico corado pelo HE, Grocott, Fite Faraco e Ziehl Nielsen; exames micológicos diretos para detec- 
ção de micoses superficiais, acrescidos de culturas em meio de Sabouraud quando havia suspeita de micoses profundas; pesquisa direta de bactérias e de micobactérias, tendo sido também utilizado cultivo em meio de Löwenstein. Tais exames foram realizados pelos Serviços de Dermatologia, Anatomia Patológica e Laboratório de Análises Clínicas do referido hospital.

As dermatoses foram classificadas em fúngicas, virais, eritêmato-escamosas, pápulo-pruríticas, induzidas por drogas, neoplásicas e outras.

\section{Resultados}

A idade dos pacientes variou de 17 a 65 anos, com média de 32,8 anos; 71\% tinham entre 20 e 39 anos (Figura I).

Dentre os 55 pacientes, 43 (78,2\%) eram do sexo masculino e $12(21,8 \%)$ do feminino. Em relação aos grupos de risco, houve predominância de heterossexuais $(56,4 \%)$, sendo $34,6 \%$ do sexo masculino e $21,8 \%$ do sexo feminino. Usuários de drogas corresponderam a 18,2\%; bissexuais a 12,7\%. Apenas 3,6\% eram homossexuais masculinos. Não se caracterizou o grupo de risco em $9,1 \%$ dos casos estudados. Não houve caso de transmissão por via transfusional. Em 23,6\% dos pacientes, havia suspeita clínica de infecção pelo HIV devido a doenças oportunistas na pele. $\mathrm{O}$ tempo entre o diagnóstico da infecção pelo HIV e a apresentaçãa da dermatose que motivou a consultafoi de atétrês anos, sendo, emmédia, de um ano e meio (Figura 2).

Foram diagnosticadas 116 dermatoses, com predominância das fúngicas e virais ( $(T a-$ bela I). A maioria dos pacientes $(67,2 \%)$ apresentou mais de uma afecção.

Os doentes estudados apresentavam ainda outras doenças e/ou alterações relacionadas ao HIV. (Tabela 2).

As manifestações cutâneo-mucosas apresentaram-se isoladas ou associadas as

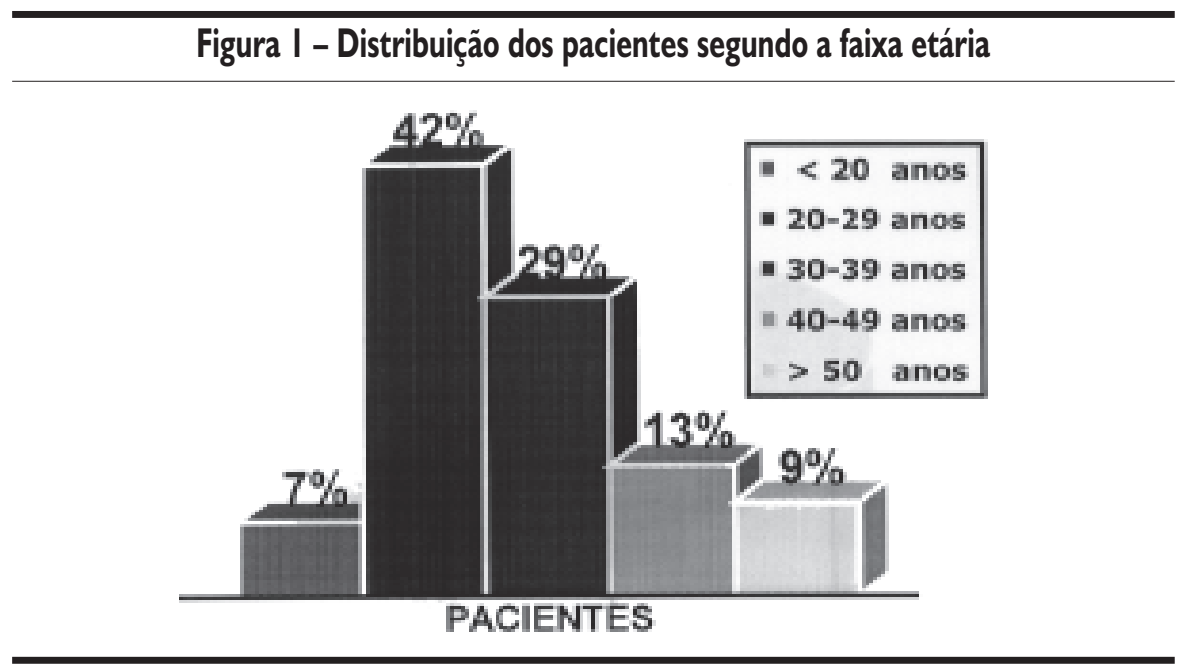

Figura 2 - Distribuição dos pacientes quanto ao tempo entre 0 diagnóstico da infecção pelo HIV e a apresentação da dermatose

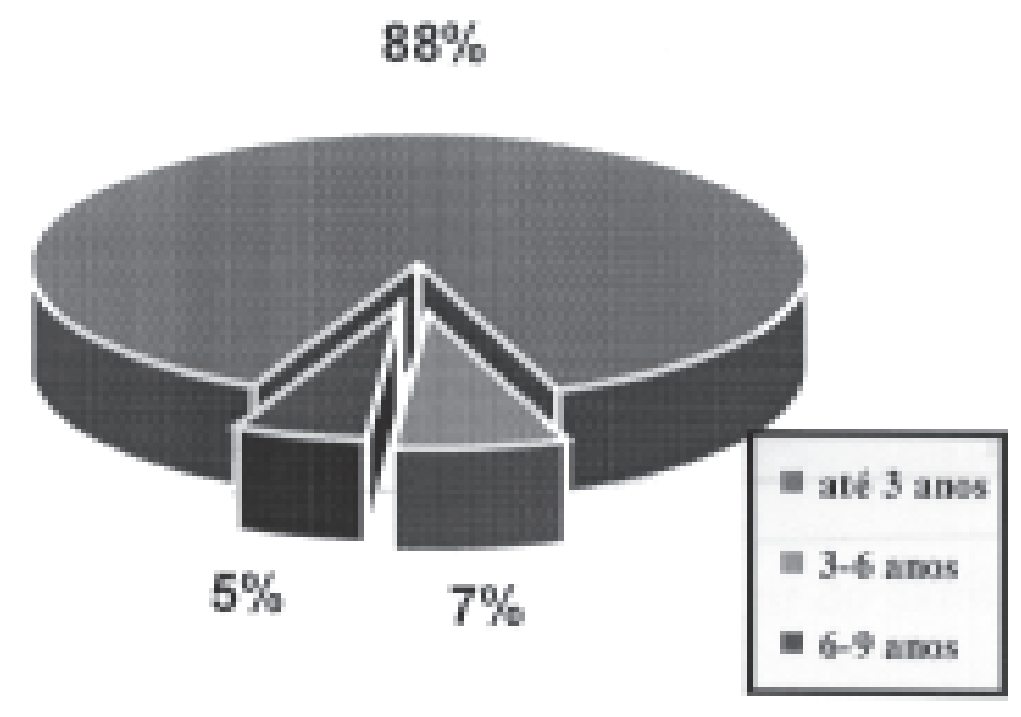

doenças relacionadas à Aids. Dos 55 pacientes, 13 (23,6\%) procuraram atendimento devido às dermatoses; a partir do caráter oportunista das lesões, aventou-se a presença do HIV. Outros estavam internados para tratamento de infecções sistêmicas e portavam lesões cutâneas e/ou mucosas que levantaram suspeitas de estarem relacionadas à imunossupressão adquirida.

\section{Discussão}

\section{Dermatoses fúngicas}

Praticamente todos os pacientes HIVpositivos apresentam infecção por Candida sp em alguma fase da doença. A candidíase pode clinicamente manifestar-se por lesões orais e também como intertrigo, queilite angular, balanopostite, paroníquia e onicomicose $^{12,25}$. É considerada um dos sinais car- 


\begin{tabular}{l}
\multicolumn{1}{c}{ Tabela I - Dern } \\
\hline A - Fúngicas \\
Candidíaseoral \\
Dematofitoses* \\
Histoplasmose \\
Candidíase(exceto oral \\
Pitiríaseversicolor \\
Cniptococose \\
TOTAL \\
B - Virais \\
Herpessimples \\
Leucoplasiapilosa \\
Herpeszoster \\
Moluscocontagioso \\
Condilomaacuminado \\
Verrugavulgar \\
TOTAL
\end{tabular}

\section{C - Eritêmato-Escamosas}

Xerodermia

Dermatiteseborréica

Psońase

TOTAL

\section{D - Pápulo-Pruríticas}

Erupçãopápulo-pruntitica

Líquen plano

TOTAL

\section{E - Induzidas por drogas}

Erupçãofixamedicamentosa

Eritemapolimorfo

Urticáriacrônica

Erupcãoacnefforme

TOTAL

\section{F - Neoplásicas}

SarcomadeKaposi

Melanomamaligno

TOTAL

\section{G - Outras Dermatoses \\ Dermatitecrônicainespećfíca \\ Vasculiteleucocitoclástica \\ Pediculose \\ TOTAL}

*Onicomicose (6), tinha crural (2), tinha do corpo ( ) etinha do pé(I)

**intertrigo (I), balanopostite (I) equeilite angular (I) dinais da imunodeficiência quando as lesões são extensas e persistentes ${ }^{26}$.

No presente estudo, a candidíase foi a dermatose mais encontrada (43,6\%). Em 21 (87,5\%) dos 24 pacientes com a infecção, havia acometimento oral. Os demais casos foram um de balanopostite, um de queilite angular e um de intertrigo. A candidíase oral é uma manifestação de alta prevalência em HIV-positivos, com frequiência variável entre $25 \%$ e $90 \%$, $15,6,8,9,13,1,4,18,19,20$. A presença de candidíase oral pode ser um indicativo de desenvolvimento subseqüente da síndrome ${ }^{25}$ e, conseqüentemente, de outras infecções oportunísticas ${ }^{26}$.

Dermatofitoses constituíram a segunda manifestação fúngica mais freqüente, sendo observadas em 18,2\% dos casos estudados. Clinicamente, apresentam-se, em geral, com características especiais, como o comprometimento de áreas extensas, evolução mais rápida e necessidade de tratamento sistêmico ${ }^{12}$. A prevalência das dermatofitoses em diferentes trabalhos varia de $3,6 \%$ a $84 \% \%^{1,5,6,9,18}$, predominando as tinhas dos pés, crural, do corpo e das unhas ${ }^{12}$. Os achados desta pesquisa enquadram-se nos dados de literatura acima referidos.

Aproximadamente 10\% dos doentes com histoplasmose disseminada apresentam lesões cutâneas ${ }^{2,23,24}$, principalmente durante afase de imunossupressão grave ${ }^{6,12}$. As lesões cutâneas generalizadas são variáveis e inespecíficas 6 ,19,22-24. Na casuística de Borges et al. ( $(1997)^{27}$, em 22,2\% dos casos ocorridos em pacientes infectados pelo HIV, a histoplasmose foi a primeira manifestação da doença, tendo sido, portanto, a infecção que definiu o diagnóstico de Aids. No presente estudo, a histoplasmose cutâneomucosa ocorreu em freqüência considerável $(10,9 \%)$, acometendo seis pacientes; destes, quatro tinham comprometimento sistêmico, com hemocultura e mielocultura positivas. Apresentaram pápulas, placas e ulcerações disseminadas em pele e/ou

Rev Assoc Med Bras 2002; 48(1): 36-41
$7,2 \%$ 


\begin{tabular}{|c|c|c|}
\hline Achados correlatos & $\mathrm{N}^{\circ}$ de Doentes & Percentual \\
\hline Anemia & 20 & $36.4 \%$ \\
\hline Meningoencefallites & 19 & $34,5 \%$ \\
\hline Diarréilalnespecífica* & 18 & $32,7 \%$ \\
\hline Tuberculose & 15 & $27,3 \%$ \\
\hline Linfopenia & 15 & $27,3 \%$ \\
\hline Candidíase(Esofágicae Disseminada) & 14 & $25,5 \%$ \\
\hline Trombocitopenia & II & $20,0 \%$ \\
\hline Pneumonias & 10 & $18,2 \%$ \\
\hline Estrongiloidíase & 6 & $10,9 \%$ \\
\hline Histoplasmose Disseminada & 6 & $10,9 \%$ \\
\hline Hepatites & 6 & $10,9 \%$ \\
\hline NeuropatiaPeriférica & 3 & $5,4 \%$ \\
\hline Linfadenomegalia & 3 & $5,4 \%$ \\
\hline Citomegalovirose & 2 & $3,6 \%$ \\
\hline Septicemia & $\mid, 81$ & $1,8 \%$ \\
\hline *Sem Agente Causal Identificado. & & \\
\hline
\end{tabular}

mucosas. $\bigcirc$ diagnóstico dermatológico foi feito através da suspeita clínica, biopsia e cultura positiva para Histoplasma capsulatum. Um dos pacientes apresentou recidiva II meses após o primeiro episódio, apesar do tratamento com anfotericina $B$, confirmando a opinião de alguns autores de que a doença é geralmente mais grave nos doentes com Aids'22.

As manifestações cutâneas da criptococose ocorrem em menos de 10\% dos pacientes com infecção sistêmica ${ }^{12,23}$. Em geral, a criptococose cutânea se manifesta como celulite, paniculite, vasculite, ulcerações, erupção acneiforme, pápulas, pústulas, placas vegetantes, abscessos e púrpura palpável| ${ }^{9,28,29}$, predominando na face e no tronco. Na presente casuística observou-se criptococose cutânea somente em um paciente que apresentava lesões papulares na face, com aproximadamente $0,5 \mathrm{~cm}$ de diâmetro e centro exulcerado, indolores, associadas à criptococose do sistema nervoso central, à neurotoxoplasmose e à histoplasmose disseminada.

\section{Dermatoses Virais}

O herpes simples é muito freqüente nos indivíduos HIV-positivos ${ }^{39,40}$ e, dependendo do grau de imunossupressão, pode causar dificuldade diagnóstica'2 ${ }^{2}$. No início da doença, as lesões mais comuns são vesículas agrupadas, semelhantes às observadas no indivíduo imunocompetente. Com a diminuição da imunidade, as lesões tornam-se ulceradas, grandes, dolorosas e disseminadas pelo tegumento ${ }^{12,23,41,43}$. Dentre os oito casos de herpes simples deste estudo, dois manifestaram-se clinicamente como vesículas agrupadas no foco de origem e esparsas pelo corpo, com duração prolongada; nos seis restantes, as vesículas localizavamse nas regiões genital, labial, nasal e/ou orofaríngea.

$\mathrm{Na}$ leucoplasia pilosa oral ocorrem lesões infiltrativas e esbranquiçadas na face lateral da língua, correlacionadas à presença do vírus Epstein Barr³3. Esta afecção é considerada um indicador sensível e possivelmente espećíico da infecção pelo HIV e altamente preditiva para o desenvolvimento
daAids ${ }^{15,34-38}$. Nesta casuística, a leucoplasia pilosa oral ocorreu em quatro pacientes, com idades e práticas sexuais variadas, todos com infecções oportunistas à época do diagnóstico.

Nos portadores do HIV, o herpes zoster apresenta-se com maior freqüência num dermátomo, mas pode surgir em vários deles, recorrer no mesmo trajeto nervoso ou estar disseminado ${ }^{41,42,43,44}$. A forma disseminada é uma manifestação freqüente na Aids, recidivante egeralmente relacionada ao declínio do estado imunológico ${ }^{12}$. Entretanto, Buchbinder et al. ${ }^{49}$ acreditam que o herpes zoster não constitui sinal fidedigno de imunodeficiência profunda, já que pode ocorrer em qualquer estágio da doença pelo HIV. Nos três casos observados de herpes zoster, as vesículas apresentaram-se agrupadas, porém distribuídas em mais de um dermátomo.

O molusco contagioso é uma infecção viral causada por um poxvírus, habitualmente auto-limitada e com predomínio na infância. Nos pacientes com imunossupressão adquirida, as lesões do molusco contagioso se caracterizam, após vários meses de evolução, pelo aumento dramático em número e tamanho $0^{8,14,19}$ e pela localização na face e região genita ${ }^{8,19,23}$. A freqüência observada neste estudo foi de $5,5 \%$ enquanto que em outros variou de $5 \%$ a $20 \%$, $1,5,6,8,9,13,23$.

\section{Dermatoses Eritêmato-Escamosas}

A xerodermia, segundo alguns autores ${ }^{14,30}$, é muito freqüente na fase avançada daAids; porém, em diferentes trabalhos, sua freqüência é variável de 3\% a 75\% 1,5,6, 24 . Acredita-se que sua etiologia se deva à desnutrição, cronicidade da doença, higiene precária ou ao próprio déficit imunológico ${ }^{12,24}$. Nesta casuística, dos 55 casos estudados, oito (I4,5\%) apresentaram xerodermia.

A dermatite seborréica ocorreu em 10,9\% dos pacientes $\mathrm{VIH}$-positivos examinados, apresentando-se no couro cabelu- 
do, na face e parte superior do tórax. $\mathrm{Na}$ literatura, observam-se freqüências de $7,4 \%$ a $83 \% 1,2,5,6,8,9,18,19$.

Dos 55 pacientes, um $(1,8 \%)$ apresentou psoríase. Tratava-se de um quadro de psoríase em placas, de moderada intensidade, comprometendo as regiões cubitais, joelhos e dorso. A prevalência da psoríase nos pacientes HIV-positivos varia na literatura de I,3\% a 8,3\% I, 14,24,46, havendo controvérsias quantoàsuaelevaçãoem relaçãoàpopulaçãogeral. Entretanto, podehaver progressão acelerada das lesões da psoríase nestes pacientes, além da possibilidade de aumento da prevalência daartrite psoriásica ${ }^{24,46}$.

\section{Dermatoses Pápulo-Pruríticas}

Na presente pesquisa houve sete casos (12,7\%) de erupção pápulo-prurítica (EPP). Tal freqüência coincide com a de outros autores, cujos percentuais variaram de I I,4\% a 48\% 12,19,22. As lesões eram geralmente pruriginosas, manifestando-se clinicamente como pápulas de 2 a $5 \mathrm{~mm}$ de diâmetro, de cor semelhante à da pele normal, não coalescentes, localizadas em cabeça, pescoço e região superior do tronco.

A EPP da Aids é uma afecção cutânea crônica caracterizada pela erupção de pápulas eritematosas e pústulas, podendo constituir a manifestação inicial nos pacientes infectados pelo HIV 45,47. Provavelmente, representa um espectro de doenças, incluindo a foliculite eosinofílica associada ao HIV, que têm achados clínicos e histológicos similares ${ }^{48}$.

\section{Dermatoses Induzidas por Drogas}

As farmacodermias ocorrem com rela-

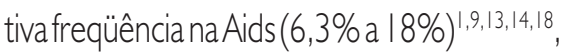
uma vez que os pacientes HIV-positivos fazem uso de grande número de medicamentos. Manifestam-se, principalmente, por hiperpigmentação, exantema, quadro urticariforme e bolhas. Na maioria das vezes, relacionam-se ao uso de sulfas e amo$x^{2} c_{i l i n a}{ }^{12,18,22}$. Neste estudo ocorreram em seis dos 55 pacientes analisados (10,9\%): eritema pigmentar fixo em três casos; eritema polimorfo, urticária crônica e erupção acneiforme, um caso de cada.

\section{Dermatoses Neoplásicas}

A presente pesquisa mostrou número reduzido de dermatoses neoplásicas. 0 sarcoma de Kaposifoi observado em apenas quatro doentes $(7,3 \%)$, com idades entre 25 e 34 anos. Um era homossexual, outro bissexual, e os dois restantes heterossexuais. Três dos quatro pacientes (75\%) apresentavam infecções oportunistas. $\mathrm{O}$ achado de baixa freqüência do sarcoma de Kaposi nesta casuística talvez seja devido ao pequeno número de homo e bissexuais estudados. O sarcoma de Kaposi é o tumor mais comum em pacientes com Aids ${ }^{12,14}$. $\mathrm{Na}$ forma clássica, as lesões se iniciam simetricamente nas mãos e nos pés, podendo envolver grandes áreas ${ }^{31}$ com placas enódulos violáceos, que podem ulcerar ${ }^{31,32}$.

O melanoma maligno foi observado em apenas um caso, equalquer associação entre melanoma e infecção pelo HIV parece representar coincidência, não tendo relação com a imunossupressão $0^{8,34}$.

\section{ConcLusões}

As dermatoses nos portadores do HIV manifestam-se de forma atíica em grande número de doentes, impondo propedêutica bem elaborada para um diagnóstico preciso.

De acordo com o tempo de descoberta do HIV, observou-se que o exame dermatológico e a biopsia de pele colaboraram no diagnóstico precoce da Aids.

Confirmou-se a maior frequêencia das dermatoses fúngicas, seguidas pelas virais nos pacientes HIV-positivos. A candidíase oral foi a dermatose mais encontrada, confirmando sua alta freqüência nestes doentes.

Algumas das dermatoses diagnosticadas, especialmente a histoplasmose, refletiram seu caráter endêmico em nossa região, tendo sido registradas em percentual mais elevado do que aqueles observados principalmente na literatura internacional.

\section{SUMMARY}

\section{SKIN DISEASES IN ACQUIRED IMMUNO- DEFICIENCY SYNDROME (AIDS): ANALYSIS OF 55 BraZiluan CASES From UberLÂNDIA - MG, BRAZIL}

Fifty-five patients with acquired immunodeficiency syndrome (AIDS) seen at the Dermatology outpatient clinic and who had mucocutaneous diseases were studied. These diseases, some of them opportunistic, are common but difficult to diagnose given the atypical features of the lesions.

OBIECTVES. The aim of this study was to analyse the frequency and clinical presentation of dermatoses related to Aids seen at the Dermatology outpatient clinic.

Methods. Fifty-five patients with Aids and mucocutaneous lesions were examined from 1995 to 1997 in a cross-sectional study carried out at the Dermatology outpatient clinic of the Universidade Federal de Uberlândia (Minas Gerais, Brazil). Biopsies and cultures were undertaken for laboratory diagnosis.

Results. One hundred sixteen dermatoses were diagnosed. Fungal infections (78\%) were the commonest among them, followed by viral infections (40\%), papulosquamous disorders (27\%), papular eruptions (18\%), adverse drug reactions (10\%), tumors (9\%) and a variety of others (7\%). The majority of the patients (67\%) had more than one type of skin disorder.

Conclusions. In agreement with previous literature data fungaland viralinfections were confirmed as the most frequent skin disorders in HIV-positive patients. Dermatological examination, laboratory tests and skin biopsy for histopathological study are necessary for appropriate diagnostic investigation of HIV-related mucocutaneous diseases considering that atypical presentation occurs in a large proportion of the patients. [Rev Assoc Med Bras 2002; 48(I): 36-4I]

KEY WORDS: Aids. Skin diseases. 


\section{REFERÊNCIAS}

I. Smith KJ, Skelton HG, Yeager J, Ledsky R, Mc Conty W, Baxten D, et al. Cutaneous findings in HIV-I-positive patients: a 42month prospective study. Military Medical Consortium for the Adwancement of Retroviral Research CCMCARR. I Am Acad Dermatol 1994; 31:746-54.

2. Kaplan MH, Sadick N, McNutt S, Meltzen M, Sarngadharam MG, Pahwa S, et al. Dermatologic findings and manifestations of acquired immunodeficiency syndrome (AIDS). I Am Acad Dermatol 1987; 16: 485-506.

3. Myskowski PL, Ahkami R. Dermatologic complications of HIV infection. Med Clin North Am 1996; 80:1415-35

4. Memar O, Cirelli R, Lee P, Tyring SK. Cutaneous manifestations of HIV-I infection. J Am Podiatr Med Assoc 1995; 85:362-74.

5. Mendel BR,Janier M, Gerbaka J, Reynau D, Menn B. Dermatologic findings in HIVinfected patients: a prospective study with emphasis on CD4+ cell count. Dermatologic 1996; 192:325-8

6. Coldiron BM, Bergstresser PR. Prevalence and clinical spectrum of skin disease in patients infected with human immunodeficiency virus. Arch Dermatol 1989; 125:357-61.

7. Abraham A. AIDS in the tropics. Dermatol Clin 1994; |2:747-51.

8. Tschachler E, Bergstresser PR, Stingl G. HIV-related skin diseases. Lancet 1996; 348:659-63.

9. Goldstein B, Berman B, Sukenik E, Frankel S]. Correlation of skin disorders with CD4 lynphocyte counts in patients with HIV/AIDS. J Am Acad Dermatol 1997; 36:262-4.

10. Walker BD. Imunologia relacionada à AIDS/SIDA. HIV e a síndrome da imunodeficiência adquirida. In: Bennett JC, Plum F, editores. Tratado de medicina interna. 2a ed. Rio de Janeiro: Guanabara Koogan, 1997. p.2027-88,

I ।. Berger TG. Dermatologic care in the AIDS patient: a 1990 update. In: Sande MA, Volberding PA, editors. The medical management of AIDS. Philadelphia: W. B. Saunders; 1990. p. ||4-30.

12. Mattos RA. Manifestações cutâneas da SIDA. In: Mattos RA, editor. Dermatologia para o clínico. São Paulo: Lemos: 1997. p.255-77

13. Katz DR, Manini MIP, Takahashi MDF. Manifestações cutaneomucosas em doentes de AIDS. An Bras Dermatol 1986; 61:19-21.

I4. Rosatelli JB, Machado A, Roselino AM. Dermatoses among Brazilian HIV-positive patients: correlation with the evolutionary phases of AIDS. Int J Dermatol 1997; 36:729-34.

15. Kwan TH, Hood AF. Associated cutaneous diseases. In: Pathology of AIDS and infection. Philadelphia: W.B. Saunder; 1992. p.|48-73.

16. Coopman SA, Johnson RA, Platt R, Stem RS. Cutaneous disease and drug reactions in HIV infection. N Engl J Med 1993; 328:1670-4.

17. Aftergut K, Cockerell C). Update on the cutaneous manifestations of HIV infection. Dermatol Clin 1999; 17:445-71.
18. Oliveira MMM, Veiga RG, Sereno AB, Manela M. Pereira Jr AC. Síndrome de imunodeficiência adquirida. Lesões cutâneas. An Bras Dermatol 1988; 63:63-6.

19. Warner LC, Fisher BK. Cutaneous manifestations of the acquired immunodeficiency syndrome. Int J Dermatol 1986; 25:337-50.

20. Yamada CK, Bohnenstengel E, Mendes AVTO, Sabongi VPG, Meira MCAM. Incidência de dermatofitoses e candidoses em pacientes HIV soropositivos. An Bras Dermatol 2000; 75:I57-63.

21. Walker MM, Griffiths CEM, Weber J. Dermatological conditions in HIV infection. Br Med J 1987; 294:29-32.

22. Azulay MM, Oliveira MALS, Assis TL, Pereira Ir AC. Micoses oportunísticas na síndrome da imunodeficiência adquirida. An Bras Dermatol 1989; 64:253-5.

23. Castano-Molina C, Cockerell C). Diagnosis and treatment of infectious diseases in HIV-infected host. Dermatol Clin 1997: 15:267-83.

24. Dover JS, Johnson RA. Cutaneous manifestations of human immunodeficiency virus infection. Arch Dermatol 1991; 127:1549-58.

25. Greenspan BDS, Greenspan JS. Oral manifestations of AIDS in dermatologic clinics of mucous membranes. Dermatol Clin 1987; 5:733-7.

26. Klein RS, Harris CA, Butkus-Small C, Moll B, Lesser M, Friedland G. Oral candidiasis in highrisk patients as the initial manifestation of the acquired imunodeficiency syndrome. N Engl J Med 1984; 311:354-8.

27. Borges AS, Ferreira MS, Silvestre MTA, Nishioka AS, Rocha A. Histoplasmose em pacientes imunodeprimidos: estudo de 18 casos observados em Uberlândia, MG. Rev Soc Bras Med Trop 1997; 30:119-24.

28. Borton LK, Wintroub BU. Disseminated cryptococosis presenting as herpetiform lesions in a homossexual man with acquired immunodeficiency syndrome. J Am Acad Dermatol 1984; 10:387-90.

29. Lynch DP, Naftolin LZ, Oral Cryptococcus neoformans infection in AIDS. Oral Surg Oral Med Oral Pathol 1987; 64:449-53.

30. Farthing CF, Staughton RCD, Rowland Payne CME. Skin disease in homosexual patients with acquired immune deficiency syndrome (AIDS) and lesser forms of human $T$ cell leukemia virus (HTLV-III) disease. Clin Exp Dermatol 1985: 10:3-12.

31. Martin J. Acquired immunodeficiency syndrome (AIDS) and Kaposi's sarcoma. Int J Dermatol 1984; 23:483-6.

32. Stewart WD, Danto JL, Maddin S. Diagnosis and treatment of cutaneous disorders. In: Dermatology. 4th ed. St. Louis: CV Mosby; 1978. p.549.

33. Greenspan JS, Greenspan D, Lennette ET, Abram DI, Conant MA, Petersen V, et al. Replication of Epstein-Barr virus within the epithelial cells of oral "hairy" leukoplakia, an AIDS-associated lesion. N Engl | Med |985; 3|3:|564-7|

34.Alesi E, Berti E, Cusini M. Oral hairy leukoplakia. I Am Acad Dermatol 1990; 22:79-86.
35. Greenspan D, Greenspan JS, Hearst NG, et al: Oral hairy leukoplakia: human immunodeficiency virus status and the risk for developing AIDS. J Infect Dis 1987; I 55:475-8I.

36. Lupton GP, James WD, Redfield RR, Brown C, Rodman OG. Oral hairy leukoplakia: A distinctive marker of human T-cell lymphotropic virus type III (HTLV-III) infection. Arch Dermatol 1987; 123:624-8.

37. Schiodt M, Greenspan D, Daniels TE, Greenspan JS. Clinical and histologic spectrum of hairy leukoplakia. Oral Surg Oral Med Oral Pathol 1987; 64:716-20.

38. Morfeldt-Manson L, Julander L, Nilsson B. Dermatitis of the face, yellow toe nail changes, hairy leukoplakia and oral candidiasis are clinical indicators of progression to AIDS/opportunistic infection in patients with HIV infection. Scand J Infect Dis 1989; 21:497-505.

39. Siegal FP, Lopez C, Hammer GS, Brown AE, Komfeld SI, Gold J, et al. Severe acquired imunodeficiency in male homossexuals, manifested by chronic perianal ulcerative herpes simplex lesions. N Eng| | Med 1981; 305:|439-44.

40. De Maubeuge J, Mascart-Lemone F, Clumeck $N$. Acquired immunodeficiency manifested as severe genital herpes. Two cases (abstract). Dermatologica 1984; 168:105-11.

4I. Quinnan GV, Masur H, Rook AH, Armstrong G, Frederick WR, Epstein J, et al. Herpesvirus infections in the acquired immunodeficiency syndrome. JAMA 1984: 252:72-7.

42.Johnson RA. Cutaneous manifestations of human immunodeficiency virus disease. In: Fitzpatrick's dermatology in general medicine. 5th ed. New York: McGraw-Hill, 1999. p.2505-38.

43.Dover JS, Johnson RA. Cutaneous manifestations of human immunodeficiency virus infec tion. Arch Dermatol 199|; |27:|383-9|.

44. Porro AM, Yoshioka MCN. Manifestações dermatológicas da infecção pelo HIV. An Bras Dermatol 2000; 75:665-9l.

45. Liautaud B, Pape JW, DeHovitz JA, Thomas F, La Roche AC, Vendier RI, et al. Pruritic skin lesions: a common initial presentation of acquired immunodeficiency syndrome. Arch Dermatol 1989; 125:629-32.

46.Jing W, Ismail R. Mucocutaneous manifestations of $H$ IV infection: a retrospective analysis of I 45 cases in a Chinese population in Malaysia. Int J Dermatol 1999; 38:457-63.

47. Boonchai W, Laohasrisakul R, Manonukul J, Kulthanan K. Pruritic papular eruption in HIV seropositive patients: a cutaneous marker for immunosupression. Int J Dermatol 1999; 38:348-50.

48. Bason MM, Berger TG, Nesbitt LT. Pruritic papular eruption of HIV-disease. Int J Dermatol 1993; 32:784-9.

49. Buchbinder SP, Katz MH, Hessol NA, Liu JY, O'Malley PM, Underwood R, et al. Herpes zoster and human immunodeficiency virus infection. J Infect Dis 1992; 166: I I53-6.

Artigo recebido: 13/09/2000

Aceito para publicação: 30/07/200 I 